\title{
Assessment of the Effect of Temporal Lobe Epilepsy on the White Matter of the Limbic System and White Matter Related to Lesions Causing Epilepsy Using Diffusion Tensor Imaging and Tractography
}

\author{
NERMEEN M.S. GARHY, M.D.*; AMR O.M.A. AZAB, M.D.*; RANIA Z. HASSAN, M.D.* and \\ ASMAA M. EBRAHEIM, M.D.** \\ The Departments of Radiology* and Neurology**, Faculty of Medicine, Cairo University
}

\begin{abstract}
Background: Diffusion tensor imaging (DTI) is one of recent advances in magnetic resonance imaging. It provides a three-dimensional (3D) localization of white matter tracts. Because there is widespread propagation of neuronal firing in seizure disorders via neuronal networks, cortical and subcortical regions including the limbic system can be affected despite a single seizure focus. DTI demonstrates diffusion changes in these regions.
\end{abstract}

Aim of Study: To detect the role of DTI and tractography techniques in the assessment of effects of temporal lobe epilepsy on the white matter of limbic system (cingulum and fornix) and white matter related to organic lesions causing epilepsy.

Patients and Methods: This is a prospective study included 40 patients diagnosed clinically as having temporal lobe epilepsy (TLE) and twenty control subjects. The patients and control groups are examined by conventional magnetic resonance imaging (MRI) followed by DTI and tractography. Tractography technique was used to draw the cingula, fornices and other white matter tracts related to the lesions causing epilepsy as well as to measure their fractional anisotropy (FA).

Results: Cingulum and fornix affection was only in the form of reduction of their FA. The affection of the white matter tracts related to organic lesions were in the form of: Reduction of their FA at the same site of the lesions, displacement by mass lesion with no signs of infiltration and missing part of the tracts in one patient with right temporo occipital area of encephalomalcia).

Conclusions: DTI and tractography technique help in assessment of the integrity of white matter tracts and could detect various pattern of their affection in patient with temporal lobe epilepsy.

Key Words: Diffusion - Tractography - Cingulum - Fornix - White Matter - Tracts - Epilepsy - Fractional anisotropy.

Correspondence to: Dr. Nermeen M.S. Garhy, The Department of Radiology, Faculty of Medicine, Cairo University

\section{Introduction}

DIFFUSION tensor imaging is a novel imaging technique that provides insight into the structural integrity of cerebral white matter through the measurement of water diffusion. It can also provide a mean to extract the three-dimensional (3D) location of white matter tracts that are difficult to precisely localize with other imaging techniques in order to study them [1]

Diffusion tensor imaging (DTl) in contrast to conventional MRI is sensitive to physiological changes that take place in the brain tissue ictally and interictally [2]

Because there is widespread propagation of synchronized neuronal firing in seizure disorders via neuronal networks, cortical and subcortical regions in the brain can be affected despite a single seizure focus. DTl and tractography have also demonstrated diffusion changes in gray and white matter tissues including subcortical structures such as the amygdala, hippocampus and thalamus ipsilateral to the seizure focus [3].

The relevance of the limbic system as a neural circuitry to TLE is well recognized. Therefore, recent DTI studies focused on their axonal integrity in TLE patients. Cingula and fornices are the two most visible white matter connections in this circuit [2]

Although DTI studies had provided insight into the extent of white matter abnormalities in TLE and had expanded our understanding of the diffuse epileptic network that is present the results have been population based and, therefore, the application of DTI as a clinical tool in TLE remains limited. Further studies, in particular longitudinal 
studies in patients with new- or recent-onset epilepsy, are necessary [4].

\section{Patients and Methods}

This study was conducted in Radio-diagnosis Department, Faculty of Medicine, Cairo University between January 2013 and December 2014. The study included 40 TLE patients including 17 Males and 23 Females, referred from neurology department and outpatient clinic. Twenty healthy individuals selected from patients relatives were assessed as a control group.

Inclusion criteria: All patients were diagnosed as having TLE with or without secondary generalization clinically. The patients were either newly diagnosed untreated or treated temporal lobe epileptics.

Exclusion criteria: Patients with other types of epilepsy partial or generalized epilepsy.

MR examination included non contrast conventional axial T1WI, coronal T2WI and coronal FLAIR on temporal lobe as well as diffusion tensor imaging medium.

Technique was performed using a standard 1.5 Tesla unit (Intera and Achiva, Philips).

Head coils were used (NV16 for Intera and NV8 and NV 16 for Achiva).

\section{Imaging parameters:}

Axial T1WI: TR 450ms, TE 15ms, Flip 69, Matrix 180x169, FOV (field of view) 210x236, Number of excitation: 2, Slice thickness: 6.0/1.5.

Coronal T2WI on temporal lobe: TR 2.2s, TE $100 \mathrm{~ms}$, Flip angle $90^{\circ}$, FOV $200 \mathrm{~mm}$, Slice thickness: 4.0/-0.01.

Coronal FLAIR on temporal lobe: TR 8.0s, TE $125 \mathrm{~ms}$, Flip angle $90^{\circ}$, FOV $200 \mathrm{~mm}$, Slice thickness: $4.0 /-0.01$

Diffusion Tensor consisted of: A single shot, spin-echo echo planar sequence in 12 encoding direction, diffusion weighting factor of $800 \mathrm{~s} / \mathrm{mm}^{2}$, TR $8000 \mathrm{~ms}$, TE $67 \mathrm{~ms}$, flip $90^{\circ}$, matrix $112 \times 110$, FOV 210x236mm, number of excitations: 2 , slice thickness: $2.0 / 00$.

All the images were transferred to the workstation (Philips extended MR workspace) supplied by the manufacturer.

Processing: Images were post-processed using software for tractography. The maps obtained were:
FA map, Directionally-encoded color FA maps, 2D and in some patients $3 \mathrm{D}$ fiber tractography.

\section{Case 1:}

Clinical data: Female patient, 30 years old, presented with seizure, started when she was 5 years old. She was diagnosed clinically as having temporal lobe epilepsy.

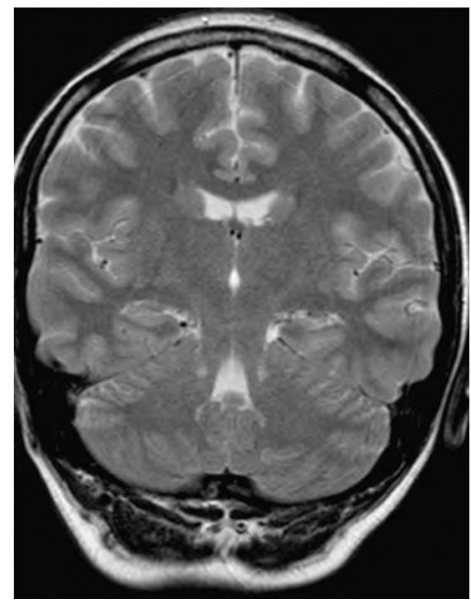

Fig. (1): Coronal T2WI image at the level of hippocampal tail showed signs of lft mesial temporal sclerosis including: Small sized left hippocampus showing bright signal intensity as compared with the right side with prominent ipsilateral temporal horn.

\section{Post processing DTI images:}
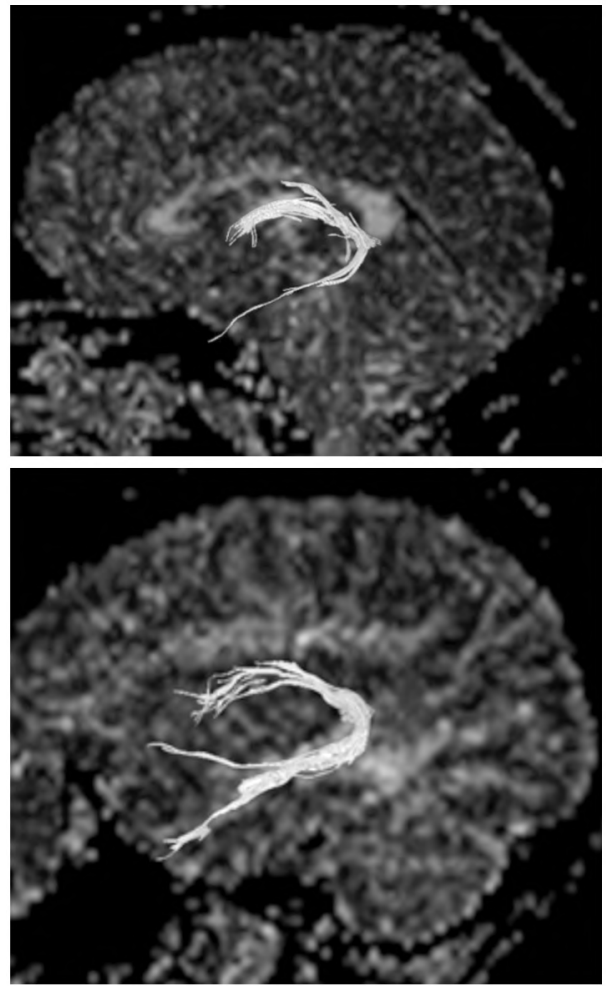

Fig. (2): Two D tractography of LT fornix (purple) and RT fornix (yellow) with measuring their FA which was 0.43 of the left fornix and 0.47 of right fornix, FA difference between both sides 0.04 . 

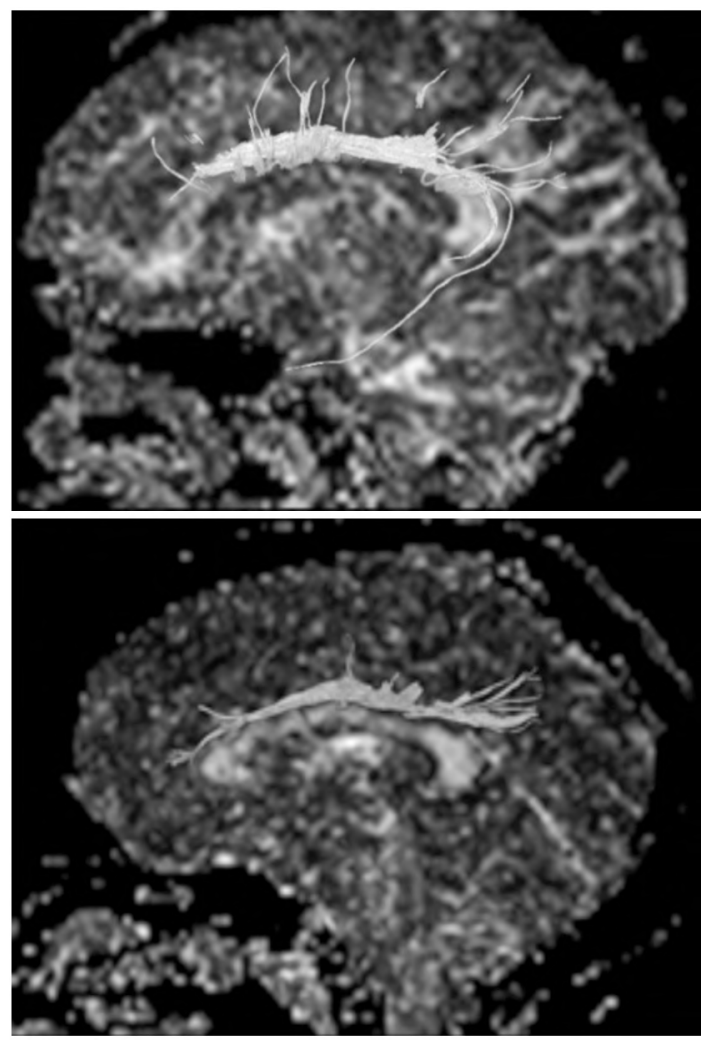

Fig. (3): Two D tractography of RT cingulum (orange) and LT cingulum (blue) with measuring their FA which was 0.527 at RT side and 0.510 on the LT side, FA difference between both sides 0.017 .

\section{Case 2:}

Clinical data: Male patient, 38 years old, presented with seizures when he was 23 years old. He diagnosed clinically as having temporal lobe epilepsy.

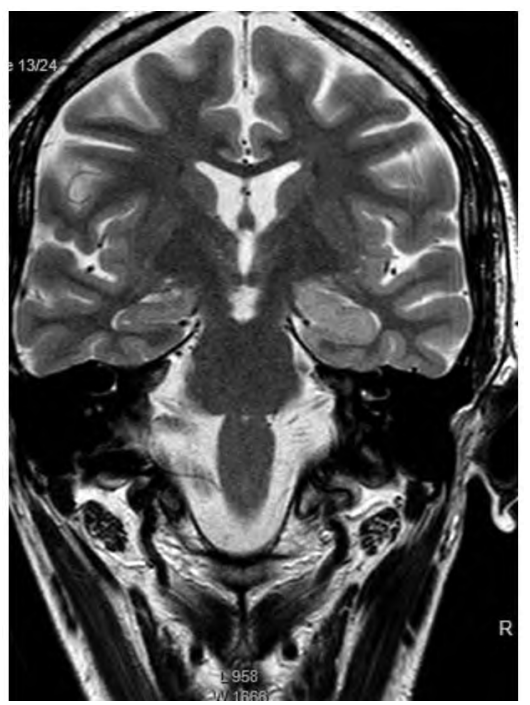

Fig. (4): Coronal T2WI (A) at the level of hippocampal head showed enlargement of the left hippocampal head eliciting bright signal intensity (arrows) as compared with the right side raising the possibility of low grade tumor of left hippocampus.

\section{Post processing DTI images:}
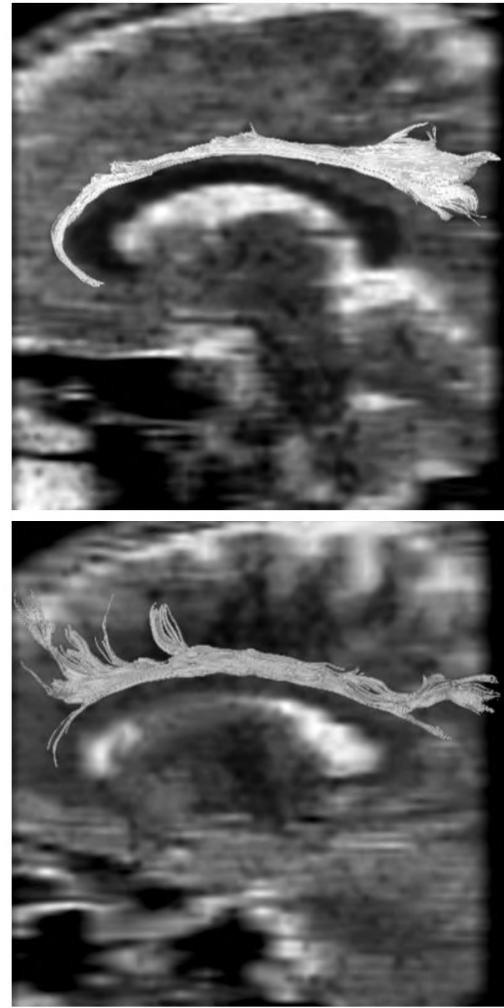

Fig. (5): Two D tractography of LTcingulum (yellow) and RT cingulum (violet) with measuring their FA which was 0.490 at LT side and 0.515 on the RT side, FA difference between both sides was 0.025 .
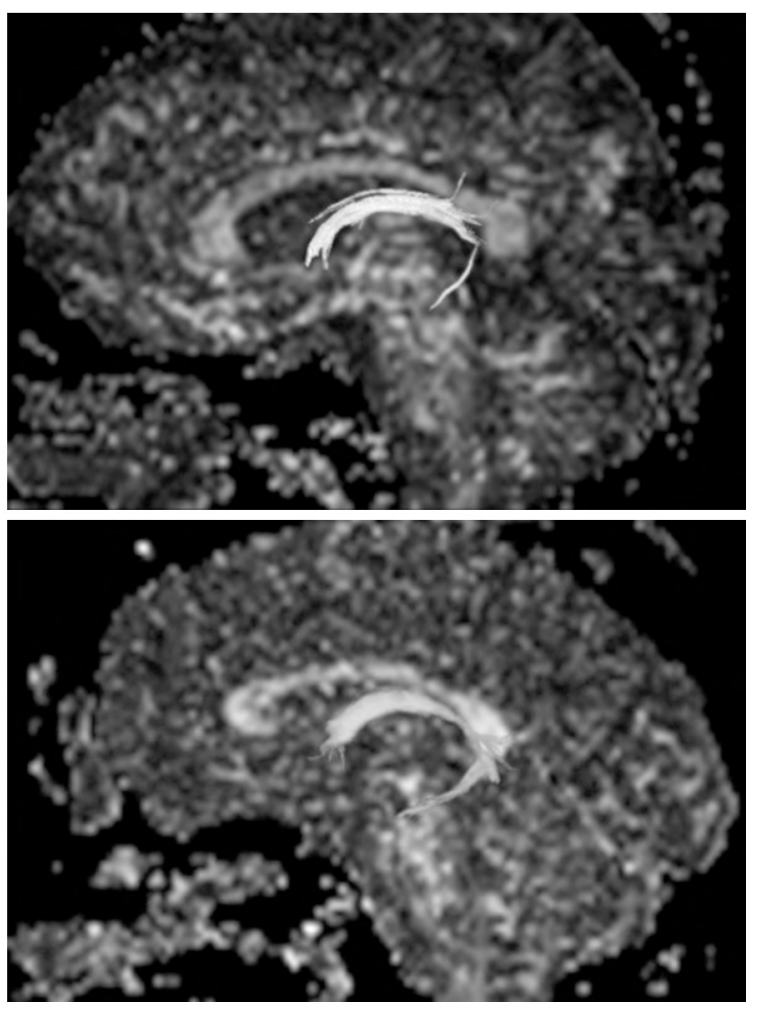

Fig. (6): Two D tractography of LT fornix (green) and RT fornix (red) with measuring their FA which was 0.464 of the LT fornix and 0.490 of RT fornix, FA difference between both sides was 0.026 . 

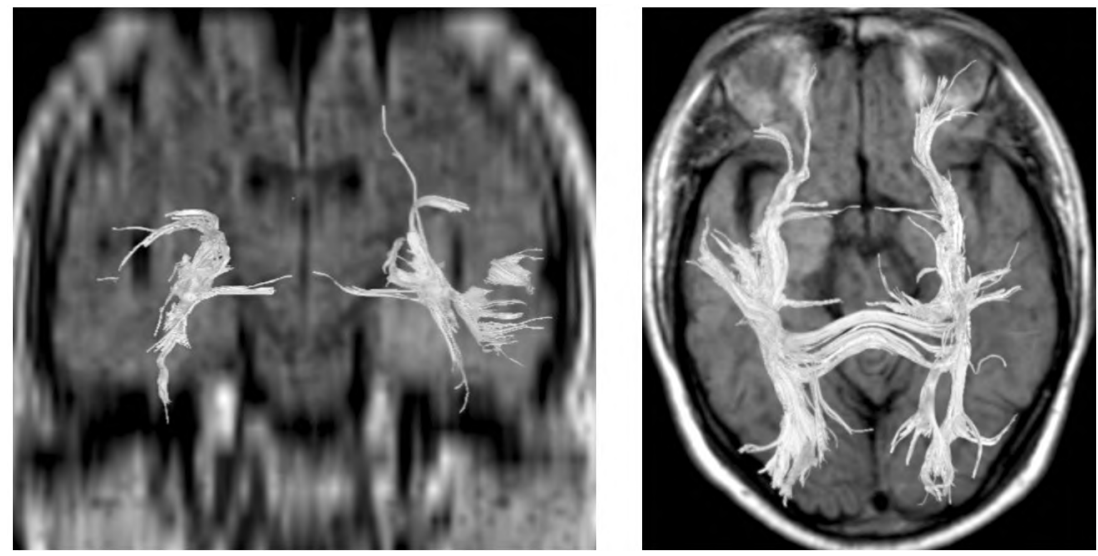

Fig. (7): Two D tractography of the related tracts, LT IFOF (inferior fronto occipital fasiculus) (green) compared with RT IFOF (purple) showing slight displacement of the left IFOF by the lesion with no evidence of its infiltration. Their FA values were measured and were 0.40 at LT side and 0.48 on the RT side.
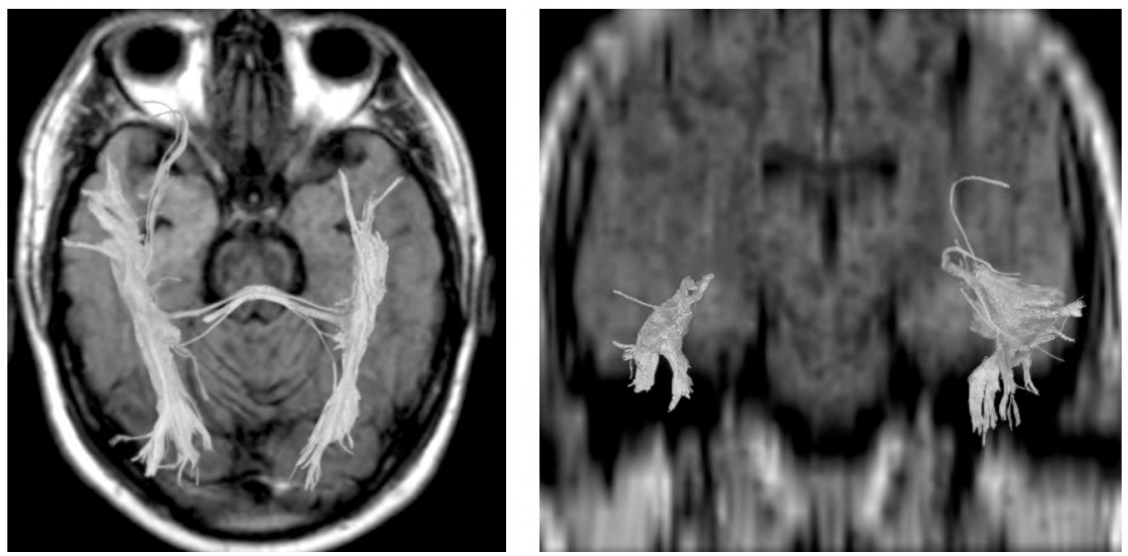

Fig. (8): Two D tractography of the related tracts, LT ILF (inferior longitudinal fasciculus) (blue) compared with RT ILF (violet) showing no signs of displacement or infiltration of LT ILF by the lesion. Their FA values were measured and were 0.438 at LT side and 0.456 at the RT side.

\section{Results}

MR examination revealed that 16 patients had signs of mesial temporal sclerosis, and twenty two patients showed no gross abnormality. The remaining two patients showed organic lesions other than MTS at the temporal lobe (Table 1).

Table (1): MR findings in patients' group.

\begin{tabular}{lcc}
\hline MR findings & Number & Percent \\
\hline No abnormality (negative cases) & 22 & 55 \\
Positive findings of MTS & 16 & 40 \\
Positive findings of other organic lesions & 2 & 5 \\
& & \\
\hline Total & 40 & 100 \\
\hline
\end{tabular}

- Data are expressed as mean \pm SD or number (\%).

- MTS = Mesial temporal sclerosis.
We studied the Effect of epilepsy on the cingula and fornices (as they are part of neural circuit in temporal lobe epilepsy). We noticed that:

Cingulum and fornix affection was only in the form of reduction of their FA ipsilateral to the epileptogenic hippocampus.

The patients were classified into positive and negative for affection of cingula and fornices by calculating mean FA differences between both cingula and fornices in the control group and demonstrating a cut off value according to which patients were classified into positive or negative for affection of cingula and fornices.

The cut off value of FA difference between both cingula was 0.016 .

The cut off value of FA difference between both fornices was 0.014 . 
We made a table and a figure that explain how we calculated the cut off value of FA difference between both cingula (Table 2, Fig. 9).

Table (2): Diagnostic indices of FA difference between both cingula in patients' and control groups using ROC curve.

\begin{tabular}{lc}
\hline & $\begin{array}{c}\text { FA difference between } \\
\text { both cingula }\end{array}$ \\
\hline Area under the roc & 0.644 \\
Cut off & $>0.016$ \\
Sensitivity & 40 \\
Specificity & 100 \\
Positive predictive value & 100 \\
Negative predictive value & 45.5 \\
\hline
\end{tabular}

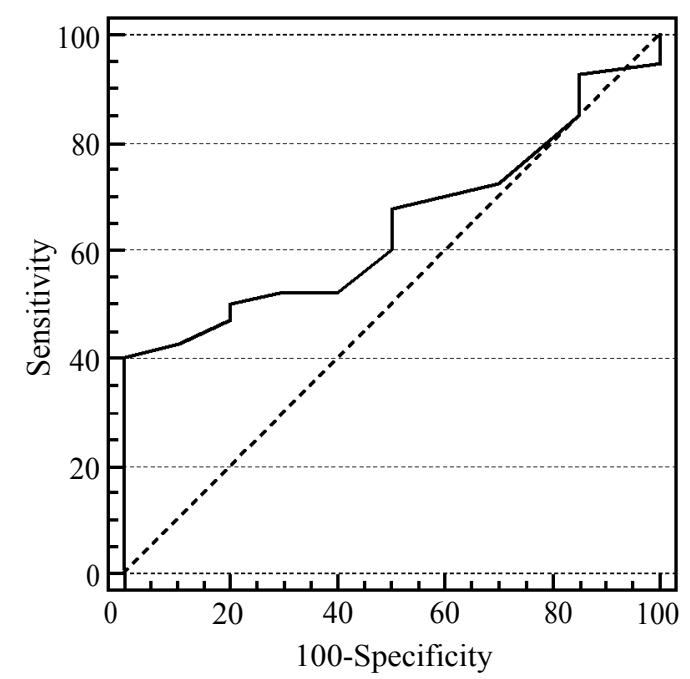

Fig. (9): ROC curve of FA difference between both cingula.

We made a table and a figure that explain how we calculated the cut off value of FA difference between both fornices (Table 3, Fig. 10 ).

Table (3): Diagnostic indices of FA difference between both fornices in patients' and control groups using ROC curve.

\begin{tabular}{lc}
\hline & $\begin{array}{c}\text { FA difference between } \\
\text { both fornices }\end{array}$ \\
\hline Area under the roc & 0.828 \\
Cut off & $>0.014$ \\
Sensitivity & 62.5 \\
Specificity & 100 \\
Positive predictive value & 100 \\
Negative predictive value & 57.1 \\
\hline
\end{tabular}

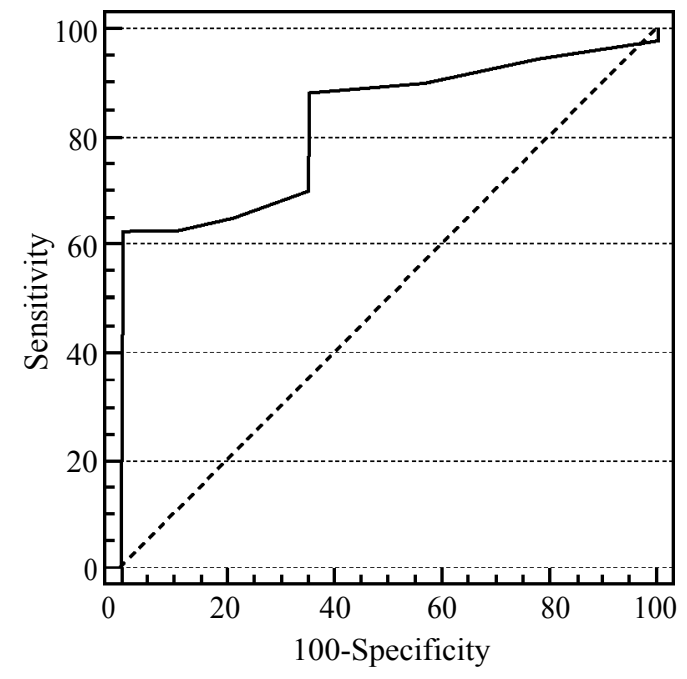

Fig. (10): ROC curve of FA difference between both fornices.

The number of TLE patients that have positive affection of the cingulum in our study was 16 $(40 \%)$ patients and the number of TLE patients that have positive affection of the fornix was 25 $(62.5 \%)$ patients.

The previous findings reflect that the fornix is more frequently affected than the cingulum in TLE patients.

We assessed the pattern of affection of the white matter tracts (inferior fronto occipital, IFOF and inferior longitudinal fasiculus, ILF) ipsilateral to the organic lesions of the temporal lobe in patients' group and we found that:

In the two patients ( $5 \%$ of patients) with structural lesions other than HS in the temporal lobe, 1 patients had areas of encephalomalcia $(2.5 \%$ of patients) and one patient (2.5\% of patients) had a mass at left temporal lobe). The affection of the white matter tracts related to these lesions (IFOF and ILF) were in the form of:

- Reduction of FA of the (IFOF and ILF) at the same site of the lesions in the all four patients.

- Displacement of (left IFOF) by mass lesion (in one case with mass in the left temporal lobe) with no signs of infiltration.

- Missing part of the (right ILF) in one patient with right temporo occipital area of encephalomalcia).

\section{Discussion}

Temporal lobe epilepsy (TLE) is the most common localization related epilepsy syndrome [5]

The ultimate goal of neuro imaging of epilepsy is not only lesion detection but also to confirm lateralization of the seizure activity to plan for a 
potential surgery as well as to provide information on etiology and patho physiologic mechanism. These goals make a potential value for new structural magnetic resonance neuro imaging technique such as DTI [6].

The aim of this study was to assess the role of DTl in the evaluation of white matter affection caused by temporal lobe epilepsy through:

- Studying the role of DTI measurement (FA) in early detection of abnormality in the white matter tract of the limbic system (as result of being part of the neural circuit in TLE) as well as of the white matter tracts related to organic lesions causing epilepsy.

- Using the tractography technique to draw the white matter tract of the limbic system (cingula and fornices) and white matter adjacent to lesions causing epilepsy to detect their interruption or infiltration.

The study was conducted on 40 patients diagnosed as having temporal lobe epilepsy, mainly clinically, and on 20 controls.

We assessed our patients using conventional MR for the presence of signs of mesial temporal sclerosis or other organic causes of epilepsy in temporal lobe like masses, areas of encephalomalcia or congenital malformation.

Several studies showed that diffusion changes in temporal lobe epilepsy not limited to the hippocampal region but it extends to involve the temporal as well as extra temporal white matters. One of these studies, the previously mentioned study done by Thivard et al., [7], they measured MD and FA values at hippocampi as well as in the white matter of the temporal lobe and extratemporal areas. They found increased diffusivity in the epileptic hippocampus (allowing its lateralization) and in the ipsilateral temporal structures associated with a decreased anisotropy along the temporal lobe, a decreased diffusivity was found in the contralateral non-sclerotic hippocampus, the amygdala, and the temporal pole, and finally, a decreased anisotropy was noted ipsilaterally in posterior extra-temporal regions. These findings support that diffusion abnormalities are not restricted to the pathological hippocampus and involve a larger network. This pattern may indirectly reflect the epileptogenic network and may be interpreted as a cause or a consequence of epilepsy.

Moreover, Liacu et al., [2] obtained DTI parametric indices including fractional anisotropy (FA) from tractography of bilateral fornix, superior and inferior cingulum fibers in 18 patients ( 9 with signs of hippocampal sclerosis and 9 without signs of hippocampal sclerosis) and 10 healthy controls. They focused on the fornix and cingulum white matter tracts since they are the major pathways involved in the epileptic network in TLE patients. On the ipsilateral seizure side, cingulum and fornix showed significant DTI indices abnormalities in both patient groups when compared with controls, most marked on the inferior cingulum. Contra lateral to the seizure side, the cingula and fornices presented significant DTI parameters abnormalities in the group with positive signs of hippocampal sclerosis when compared with controls. This goes with the finding of Liu et al., [2] who conducted a study to investigate the diffusion properties of thirteen major white matter tracts in patients with TLE with positive signs of MTS and in patients with non lesional TLE as well as in controls. They found reduced anisotropy in ten tracts for TLE with positive signs of MTS, but only the parahippocampal cingulum and tapetum for non lesional TLE. The diffusion abnormalities in tracts were observed within and beyond the temporal lobe in TLE with positive signs of MTS and were more widespread than in non lesional TLE. These findings suggest different dysfunctional networks in TLE patients with and without MTS.

The results in our study supported the conclusion of the previously mentioned studies. We used DTI parameters (FA) and tractography technique to study the effect of temporal lobe epilepsy on the tracts of the limbic system (cingula and fornices, as a part of the neural circuit involved in epilepsy).We noticed that in all patients, the cingula and fornices showed maintained integrity though their FA was reduced. Fornices were more frequently affected than cingula. This differs from the results reported by Liacu et al., [2] as they found that cingula were more frequently affected than the fornices.

In patients with organic lesions at the temporal lobe we used tractography technique to demonstrate the effect of those lesions on the related white matter tract. The patterns of affection of the white matter tracts related to those lesions (IFOF and ILF) were in the form of:

- Reduction of FA of the (IFOF and ILF) at the same site of the lesions in the all four patients with organic lesions.

- Displacement of (left IFOF) by mass lesion (in one case with mass in the left temporal lobe) with no signs of its infiltration. 
- Missing part of the (right ILF) in one patient with right temporo occipital area of encephalomalacia).

Several studies done to demonstrate the effect of different lesions on the cerebral white matter especially in cases of brain tumors. Among these studies, those done by Iliescu et al., 2011 and Ibrahim et al., 2013 [8,9], they assessed the role of tractography in evaluation of cerebral white matter in cases of brain tumors. Conventional MRI before and after contrast administration was done followed by DTI and tractography of different white matter tracts in direct relation to the tumor. The values obtained were compared to the normal unaffected tract in the contralateral side. White matter involvement by a tumor was in the form of displacement, infiltration, disruption or edema. Ibrahim et al., 2013 [9] found that the prevalence of tract displacement was higher among benign group in comparison to the malignant group, while the prevalence of disruption was higher among the malignant group, in comparison to the benign group.

\section{Conclusion:}

DTI and tractography technique represent a recent advances in MRI. They allow 3D reconstruction of the cerebral white matter, and through this we can detect any changes in the white matter integrity and direction as well as their infiltration by tumors. Temporal lobe epilepsy causes diffusion changes that extends beyond the epileptogenic hippocampus and can be detected using DTI and tractography.

\section{References}

1- DONALD W.G.: Diffusion tensor imaging in temporal lobe epilepsy. Epilepsia, 52 (Suppl. 4): 32-34, 2011.

2- LIACU D., IDY-PERETTI I., DUCREUX D., et al.: Diffusion tensor imaging tractography parameters of limbic system bundles in temporal lobe epilepsy patients. Journal of magnetic resonance imaging, 36: 561-568, 2012.

3- KIMIWADA T., JUHASZ C., MAKKI M., et al.: Hippocampal and thalamic diffusion abnormalities in children with temporal lobe epilepsy. Epilepsia , 47: 167-175, 2006.

4- GROSS D.W.: Diffusion tensor imaging in temporal lobe epilepsy. Epilepsia, 52: 32-34, 2011.

5- CONCHA L., DANIEL J.L., BEAULIEU C., et al.: In vivo diffusion tensor imaging and histopathology of fimbria-fornix in temporal lobe epilepsy. Journal of neuroscience, 30 (3): 996-1002, 2010.

6- SALEEM S. and SAID A.H.: Role of diffusion tensor Imaging in management of epileptic patients with focal brain lesions. The Egyptian Journal of Radiology and Nuc. Med., 39 (1): 269-278, 2008.

7- THIVARD L., LEHERICY S., KRAINIK A., et al.: Diffusion tensor imaging in medial temporal lobe epilepsy with hippocampal sclerosis. Neuro Image, 28: 682-690, 2005.

8- ILIESCU B., DABIJA A., FAIYAD Z., et al.: The role of MR tractography in pre surgical planning personal series of 25 cases and a review of the literature. Romanian Neurosurgery, XVIII 4: 483-490, 2012.

9- IBRAHIM A.S., GOMAA M., SAKR H., et al.: Role of diffusion tensor imaging in charectarization and pre operative planning of brain neoplasms. The Egyptian Journal of Radiology and Nuclear Medicine, 2013.

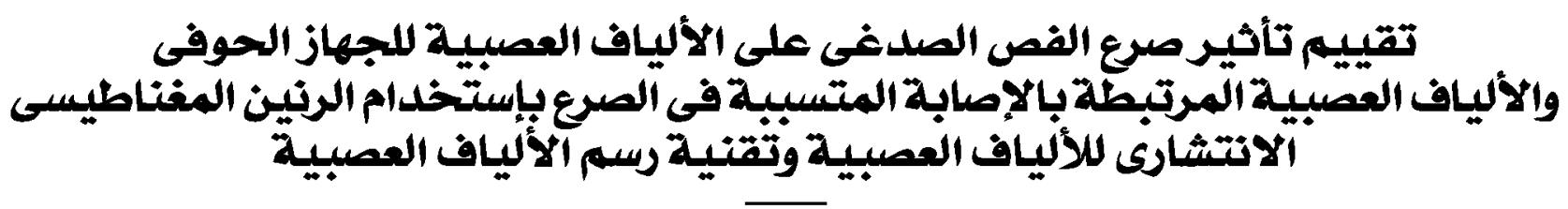

إن هذه الدراسة تمت لتقييم دود الرنين المغناطيسي الانتشارى للألياف العصبية في إكتشاف التفيرات غير الطبيعية في الألياف العصبية

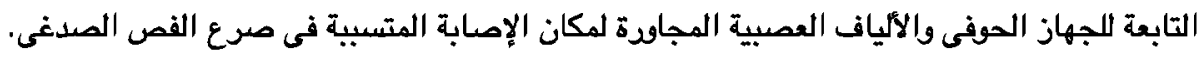

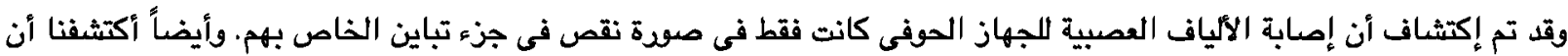

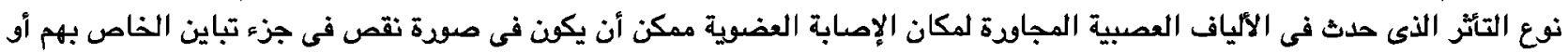
تغيير مسارهم أو فقد جزء منهم.

ونستخلص من ذلك أن الرنين المغناطيسى الانتثارى للألياف العصبية يساعدنا فى أكتشاف التفيرات غير الطبيعية فى الألياف العصبية

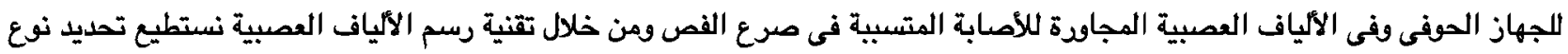
إصابة الألياف العصبية للمخ فى مرضى صفي الافع الفص الصدئ. 